\title{
Substructuring Methods in Nonlinear Function Spaces
}

\author{
Oliver Sander
}

Bericht Nr. 383

Januar 2014

Key words: substructuring, manifold-valued functions, Cosserat shells, Neumann-Neumann method

AMS Subject Classifications: 65N55, 65J15, 74K25

Institut für Geometrie und Praktische Mathematik RWTH Aachen

Templergraben 55, D-52056 Aachen (Germany)

Oliver Sander,

Institut für Geometrie und Praktische Mathematik, RWTH Aachen, Templergraben 55, 52056 Aachen, Germany, e-mail: sander@igpm.rwth-aachen.de 


\title{
Substructuring Methods in Nonlinear Function Spaces
}

\author{
Oliver Sander
}

\begin{abstract}
We generalize substructuring methods to problems for functions $f: \Omega \rightarrow M$, where $\Omega$ is a domain in $\mathbb{R}^{d}$ and $M$ is a Riemannian manifold. Examples for such functions include configurations of liquid crystals, ferromagnets, and deformations of Cosserat materials. We show that a substructuring theory can be developed for such problems. While the theory looks very similar to the linear theory on a formal level, the objects it deals with are much more general. In particular, iterates of the algorithms are elements of nonlinear Sobolev spaces, and test functions are replaced by sections in certain vector bundles. We derive various solution algorithms based on preconditioned Richardson iterations for a nonlinear Steklov-Poincaré formulation. Preconditioners appear as bundle homomorphisms. As a numerical example we compute the deformation of a geometrically exact Cosserat shell with a Neumann-Neumann algorithm.
\end{abstract}

\section{Variational Problems in Spaces of Manifold-Valued Functions}

Let $\Omega$ be a domain in $\mathbb{R}^{d}$, and $M$ a smooth, connected, finite-dimensional manifold with positive injectivity radius. We assume $M$ to be equipped with a metric $g$, which induces an exponential map exp : $T M \rightarrow M$.

Oliver Sander

Institut für Geometrie und Praktische Mathematik, RWTH Aachen, Templergraben 55, 52056 Aachen, Germany, e-mail: sander@igpm.rwth-aachen.de 


\subsection{Spaces of Manifold-Valued Functions}

In this article we consider space ${ }^{1}$ of functions $v: \Omega \rightarrow M$. We first define functions of Sobolev smoothness.

Definition 1. Let $\imath: M \rightarrow \mathbb{R}^{N}$ be an isometric embedding for some $N \in \mathbb{N}$, and let $k \in \mathbb{N}_{0}$ and $p \in \mathbb{N}$. Define

$$
W^{k, p}(\Omega, M):=\left\{v \in W^{k, p}\left(\Omega, \mathbb{R}^{N}\right): v(x) \in \imath(M) \text { a.e. }\right\},
$$

where $W^{k, p}\left(\Omega, \mathbb{R}^{N}\right)$ is the usual Sobolev space of $N$-component vector-valued functions on $\Omega$.

Note that $W^{k, p}(\Omega, M)$ does not have a linear structure. By the Sobolev embedding theorem, it is a Banach manifold if $k>d / p[9]$.

To formulate variational problems in such spaces we need to construct test functions. Unlike in linear spaces, test function spaces for a function $u: \Omega \rightarrow M$ depend on $u$.

Definition 2. Let $u \in W^{k, p}(\Omega, M)$. A vector field along $u$ is a map $\eta: \Omega \rightarrow$ $T M$, such that $\eta(x) \in T_{u(x)} M$ for almost all $x \in \Omega$.

More abstractly, vector fields along $u$ are sections in a certain vector bundle. While the concept of a vector bundle is standard (see, e.g., [6]), we state it here for completeness.

Definition 3. Let $E$ and $B$ be two differentiable manifolds, and $\pi: E \rightarrow B$ a differentiable map. The triple $(E, \pi, B)$ is called a vector bundle if each fiber $E_{x}:=\pi^{-1}(x), x \in B$ has an $n$-dimensional real vector space structure, and the following triviality condition holds. For each $x \in B$, there exists a neighborhood $U$ and a diffeomorphism

$$
\varphi: \pi^{-1}(U) \rightarrow U \times \mathbb{R}^{n}
$$

with the property that for every $y \in U \subset B$

$$
\left.\varphi\right|_{E_{y}}: E_{y} \rightarrow\{y\} \times \mathbb{R}^{n}
$$

is a bijective linear map. Such a pair $(\varphi, U)$ is called a bundle chart.

In other words, vector bundles are spaces that locally look like products $U \times$ $\mathbb{R}^{n}$. We call $E$ the total space, $B$ the base space, and $\pi$ the bundle projection of the vector bundle. The prototypical vector bundle is the tangent bundle $(T M, \pi, M)$ of a smooth manifold $M$. In this case, the bundle projection $\pi$ maps tangent vectors to their base points.

Vector bundles allow to generalize the concept of a map between spaces. A vector bundle section is an object $s$ that locally is a map $\left.s\right|_{U}: U \rightarrow \mathbb{R}^{n}$.

${ }^{1}$ We use the word space in a topologist's sense here, without implying the existence of a linear structure. 
Definition 4. Let $(E, \pi, B)$ be a vector bundle. A section of $E$ is a map $s: B \rightarrow E$ with $\pi \circ s=\operatorname{Id}_{B}$.

In particular, a map $f: \Omega \rightarrow \mathbb{R}^{n}$ can be interpreted as a section in the trivial bundle $\left(\Omega \times \mathbb{R}^{n}, \pi, \Omega\right)$. A section in the tangent bundle $T M$ of a smooth manifold $M$ is a vector field on $M$.

Let now $N$ be another smooth manifold, $f: B \rightarrow N$ a continuous map, and $(E, \pi, N)$ a vector bundle over $N$. We pull back the bundle via $f$, to obtain a bundle $f^{*} E$ over $B$, for which the fiber over $x \in B$ is $E_{f(x)}$, the fiber over the image of $x$.

Definition 5. Let $f: B \rightarrow N$ be a continuous map, and $(E, \pi, N)$ a vector bundle over $N$ with bundle charts $(\varphi, U)$. The pulled back bundle $f^{*} E$ is the bundle over $B$ with bundle charts $\left(\varphi \circ f, f^{-1}(U)\right)$.

With these preliminaries we can interpret vector fields along a continuous function as vector bundle sections.

Lemma 1. Let $f: \Omega \rightarrow M$ be continuous. A vector field $\eta$ in the sense of Definition 2 is a section in the bundle $f^{*} T M$.

So far, we have not mentioned the regularity of sections of vector bundles. The following definition is given in [6].

Definition 6. Let $(E, \pi, B)$ be a vector bundle, and $s: B \rightarrow E$ a section of $E$ with compact support. We say that $s$ is contained in the Sobolev space $W^{k, p}(E)$, if for any bundle atlas with the property that on compact sets all coordinate changes and all their derivatives are bounded, and for any bundle chart $\varphi:\left.E\right|_{U} \rightarrow U \times \mathbb{R}^{n}$ from such an atlas, we have that $\left.\varphi \circ s\right|_{U}$ is contained in $W^{k, p}\left(U, \mathbb{R}^{n}\right)$.

As a special case of this we can define vector fields of Sobolev smoothness along a given continuous function $f: \Omega \rightarrow M$.

Definition 7. Let $f: \Omega \rightarrow M$ be continuous, and $\eta$ a vector field along $f$. We say that $\eta$ is of $k, p$-Sobolev smoothness, $\eta \in \Xi_{f}^{k, p}$, if it is a $k, p$-section in the sense of Definition 6.

Finally, we need a trace theorem for vector fields along a function. We restrict our attention to $k=1, p=2$. The following is a special case of a result proved in [5]. We denote by $\mathcal{D}(\Omega, E)$ the smooth sections in $(E, \pi, \Omega)$ and by $\mathcal{D}\left(\Omega,\left.E\right|_{\Gamma}\right)$ the smooth sections of the bundle restriction on $\Gamma$.

Lemma 2. Let $\Omega$ have a $C^{\infty}$ boundary, and let $(E, \pi, \Omega)$ be a vector bundle over $\Omega$. Let $\Gamma$ be a part of the boundary of $\Omega$, and suppose it is a submanifold of $\bar{\Omega}$. Then the pointwise restriction $\operatorname{tr}_{\Gamma}: \mathcal{D}(\Omega, E) \rightarrow \mathcal{D}\left(\Gamma,\left.E\right|_{\Gamma}\right)$ extends to a linear and bounded operator from $W^{1,2}(E)$ onto $W^{\frac{1}{2}, 2}\left(\left.E\right|_{\Gamma}\right)$, i.e.,

$$
\operatorname{tr}_{\Gamma} W^{1,2}(E)=W^{\frac{1}{2}, 2}\left(\left.E\right|_{\Gamma}\right)
$$


Moreover, $\operatorname{tr}_{\Gamma}$ has a linear and bounded right inverse, an extension operator $\operatorname{Ex}_{\Omega}: W^{\frac{1}{2}, 2}\left(\left.E\right|_{\Gamma}\right) \rightarrow W^{1,2}(E)$.

For $p \neq 2, p \geq 1$ the trace operator still exists, but the traces are only contained in certain spaces of Besov type [5]. Trace theorems for functions in $W^{1, p}(\Omega, M)$ also exist (see, e.g., [7] Chap. 1.12]), but in the following we only look at continuous functions anyway.

\subsection{Variational and Minimization Problems}

We now consider variational problems in the space $W^{1, p}(\Omega, M)$. Let $\alpha$ be a form on $W^{1, p}(\Omega, M) \cap C(\Omega, M)$, i.e., for each continuous $u \in W^{1, p}(\Omega, M)$, $\alpha[u]$ is a linear map $\Xi_{u}^{1, p} \rightarrow \mathbb{R}$. We look for zeros of such a form, that is we look for continuous functions $u \in W^{1, p}(\Omega, M)$ such that

$$
\alpha[u](\eta)=0 \quad \text { for all } \eta \in \Xi_{u}^{1, p} .
$$

A bit more generally, we want to allow Dirichlet boundary conditions on part of the boundary of $\Omega$. Since for that case we need the trace theorem (Lemma 2) we restrict ourselves to $p=2$ again. Let $\Gamma_{D}$ be a subset of positive $d$-1-dimensional measure of $\partial \Omega$. For a function $u_{0}: \Gamma_{D} \rightarrow M$ sufficiently smooth define the space $H_{D}^{1}:=\left\{v \in W^{1,2}(\Omega, M) \cap C(\Omega, M): \operatorname{tr}_{\Gamma_{D}} v=u_{0}\right\}$, and for each $u \in H_{D}^{1}$ define $\Xi_{u, 0}^{1,2}=\left\{\eta \in \Xi_{u}^{1,2}: \operatorname{tr}_{\Gamma_{D}} \eta=0\right\}$. We then look for a function $u \in H_{D}^{1}$ such that

$$
\alpha[u](\eta)=0 \quad \text { for all } \eta \in \Xi_{u, 0}^{1,2} .
$$

As an example, we consider minimization problems in $W^{1, p}(\Omega, M)$. Let $J: W^{1, p}(\Omega, M) \rightarrow \mathbb{R}$ be a functional. We want to find minimizers of $J$, i.e., functions $u \in W^{1, p}(\Omega, M)$ such that

$$
J(u) \leq J(v)
$$

for all $v$ in a neighborhood of $u$.

We expect $J$ to be differentiable. By this we mean that for each curve $c:[-\epsilon, \epsilon] \rightarrow W^{1, p}(\Omega, M)$ the real function

$$
J \circ c:[-\epsilon, \epsilon] \rightarrow \mathbb{R}
$$

is differentiable. We then define the differential of $J$ at a point $u \in W^{1, p}(\Omega, M)$.

Definition 8. Let $c:[-\epsilon, \epsilon] \rightarrow W^{1, p}(\Omega, M)$ be a differentiable path with $c(0)=u$. Then $c^{\prime}(0)$ is an element of $\Xi_{u}^{1, p}$, i.e., a vector field along $u$. We define the form $d J[u]: \Xi_{u}^{1, p} \rightarrow \mathbb{R}$ by setting 


$$
d J[u](\eta):=(J \circ c)^{\prime}(0)
$$

if $c^{\prime}(0)=\eta$.

The following trivial property follows directly from the definition.

Lemma 3. For each $u, d J[u]$ is a linear form.

The first-order optimality conditions of (3) are then a variational problem of the form (1).

Lemma 4. Let $u$ be a local minimizer of $J$ in $W^{1, p}(\Omega, M)$. Then

$$
d J[u](\eta)=0
$$

for all $\eta \in \Xi_{u}^{1, p}$.

If instead $J$ is minimized in a space of functions with prescribed Dirichlet values, then a variational problem like (2) is obtained.

\section{Substructuring Formulation}

The weak problem (2) can be written as a coupled problem, consisting of two subdomain problems and suitable coupling conditions. This is well-known for linear problems in linear spaces [10, Chap. 1.2]. We show that the argument used there also holds for nonlinear function spaces.

Assume that $\Omega$ is partitioned in two nonoverlapping subdomains $\Omega_{1}$ and $\Omega_{2}$, and that the interface $\Gamma:=\bar{\Omega}_{1} \cap \bar{\Omega}_{2}$ is a $d-1$-dimensional Lipschitz manifold. We note the following technical results, which follow directly from the corresponding results for scalar-valued Sobolev spaces and Definition 1 (see also [7, Thm. 1.12.3]).

Lemma 5. 1. If $u \in W^{1, p}(\Omega, M)$, then $\left.u\right|_{\Omega_{i}} \in W^{1, p}\left(\Omega_{i}, M\right)$ for $i=1,2$.

2. Let $u_{i} \in W^{1, p}\left(\Omega_{i}, M\right)$ for $i=1,2$ and $\operatorname{tr}_{\Gamma} u_{1}=\operatorname{tr}_{\Gamma} u_{2}$. Then the function $u: \Omega \rightarrow M$ defined by

$$
u(x):= \begin{cases}u_{1}(x) & \text { if } x \in \Omega_{1} \\ u_{2}(x) & \text { if } x \in \Omega_{2}\end{cases}
$$

is contained in $W^{1, p}(\Omega, M)$.

Suppose that $\alpha$ is a linear form on $W^{1, p}(\Omega, M)$. We assume that $\alpha$ is separable in the sense that there are linear forms $\alpha_{i}$ on $W^{1, p}\left(\Omega_{i}, M\right), i=1,2$, such that

$$
\alpha[u](\eta)=\sum_{i=1}^{2} \alpha_{i}\left[\left.u\right|_{\Omega_{i}}\right]\left(\left.\eta\right|_{\Omega_{i}}\right) \quad \text { for all } u \in W^{1, p}(\Omega, M), \eta \in \Xi_{u}^{1, p} .
$$


This holds in particular if $\alpha$ is defined as an integral over a local density.

For a formal statement of our substructuring result we need the following spaces.

Definition 9. Let $u_{0}: \Gamma_{D} \rightarrow M$ be a function of prescribed Dirichlet values, of sufficient smoothness. For $i=1,2$ set

$$
H_{i}:=\left\{v_{i} \in W^{1,2}\left(\Omega_{i}, M\right) \cap C\left(\Omega_{i}, M\right):\left.v_{i}\right|_{\Gamma_{D} \cap \partial \Omega_{i}}=u_{0}\right\} .
$$

For $i=1,2$ and each $v_{i}: \Omega_{i} \rightarrow M$ continuous set

$$
V_{i, v_{i}}^{0}:=\left\{\eta_{i} \in \Xi_{v_{i}}^{1,2}: \eta_{i}(x)=0 \in T_{v_{i}(x)} M \text { for almost all } x \in \Gamma_{D} \cup \Gamma\right\} .
$$

Also, we define the interface space

$$
\Lambda:=\left\{w: \Gamma \rightarrow M \text { such that } \operatorname{tr}_{\Gamma} v=w \text { for some } v \in H_{1}\right\},
$$

and the corresponding spaces of test functions on $\Gamma$

$$
\Xi_{w}^{1 / 2}:=\Xi_{w}^{\frac{1}{2}, 2}
$$

for each continuous $w \in \Lambda$.

Note that the $V_{i, v_{i}}^{0}$ and $\Xi_{w}^{1 / 2}$ are linear spaces, whereas the $H_{i}$ and $\Lambda$ are not. Unlike in the linear case, the test function spaces are replaced by entire families of spaces, parametrized by functions $v_{i} \in H_{i}$ and $w \in \Lambda$, respectively.

Lemma 6. The weak problem (2) is equivalent to: Find $u_{i} \in H_{i}, i=1,2$, such that

$$
\begin{aligned}
\alpha_{i}\left[u_{i}\right]\left(\eta_{i}\right) & =0 & & \forall \eta_{i} \in V_{i, u_{i}}^{0}, i=1,2 \\
\operatorname{tr}_{\Gamma} u_{1} & =\operatorname{tr}_{\Gamma} u_{2} & & \\
\alpha_{1}\left[u_{1}\right]\left(\operatorname{Ex}_{\Omega_{1}} \mu\right) & =-\alpha_{2}\left[u_{2}\right]\left(\operatorname{Ex}_{\Omega_{2}} \mu\right) & & \text { for all } \mu \in \Xi_{\operatorname{tr}_{\Gamma} u_{1}}^{1 / 2}
\end{aligned}
$$

where $\operatorname{Ex}_{\Omega_{i}}, i=1,2$ is an extension operator from $\Xi_{\operatorname{tr}_{\Gamma} u_{1}}^{1 / 2}$ to $V_{i, u_{i}}^{0}$.

Note that the existence of the extension operators $\operatorname{Ex}_{\Omega_{i}}$ is ensured by Lemma 2.

Proof. We follow the argument in [10, Chap.1.2], and show first that the substructuring formulation is a consequence of (2). Let $u$ be a solution of (2). Consequently, it is an element of $W^{1,2}(\Omega, M) \cap C(\Omega, M)$, and by Lemma 5, the subdomain functions $u_{i}:=\left.u\right|_{\Omega_{i}}, i=1,2$ are in $H_{1}$ and $H_{2}$, respectively. Equation (6) follows because $u$ is continuous. Also, (5) holds, because any test function $v_{i} \in V_{i, u_{i}}^{0}$ can be extended by zero to a test function in $\Xi_{u, 0}^{1,2}$. Finally, for every $\mu \in \Xi_{\operatorname{tr}_{\Gamma} u_{1}}^{1 / 2}$ define 


$$
\operatorname{Ex} \mu:= \begin{cases}\operatorname{Ex}_{\Omega_{1}} \mu & \text { in } \Omega_{1}, \\ \operatorname{Ex}_{\Omega_{2}} \mu & \text { in } \Omega_{2},\end{cases}
$$

and note that $\operatorname{Ex} \mu \in \Xi_{u, 0}^{1}$. Therefore, $\operatorname{Ex} \mu$ is a valid test function for (2). Together with the separability (4) of $\alpha$ we get

$$
0=\alpha[u](\operatorname{Ex} \mu)=\alpha_{1}\left[u_{1}\right]\left(\operatorname{Ex}_{\Omega_{1}} \mu\right)+\alpha_{2}\left[u_{2}\right]\left(\operatorname{Ex}_{\Omega_{2}} \mu\right)
$$

which is $(7)$.

To show the other direction let $u_{i}, i=1,2$, be a solution of (5)-(7), and define

$$
u:= \begin{cases}u_{1} & \text { in } \Omega_{1} \\ u_{2} & \text { in } \Omega_{2} .\end{cases}
$$

Since $u_{1}=u_{2}$ on $\Gamma$ we can invoke Lemma 5 to obtain that $u \in W^{1, p}(\Omega, M)$; additionally, $u$ is continuous.

Let $\eta \in \Xi_{u}^{1,2}$ be a test function at $u$. By Lemma 2 it has a trace $\mu:=\operatorname{tr} \eta$ with $\mu \in \Xi_{\operatorname{tr}_{\Gamma} u}^{1 / 2}$. Then $\left(\left.\eta\right|_{\Omega_{i}}-\operatorname{Ex}_{\Omega_{i}} \mu\right) \in V_{i, u_{i}}^{0}$. With this we can compute

$$
\begin{aligned}
\alpha[u](\eta) & =\sum_{i=1}^{2} \alpha_{i}\left[u_{i}\right]\left(\left.\eta\right|_{\Omega_{i}}\right) & & \text { (by separability }(4)) \\
& =\sum_{i=1}^{2}\left[\alpha_{i}\left[u_{i}\right](\underbrace{\left.\eta\right|_{\Omega_{i}}-\operatorname{Ex}_{\Omega_{i}} \mu}_{\in V_{i, u_{i}}^{0}})+\alpha_{i}\left[u_{i}\right]\left(\operatorname{Ex}_{\Omega_{i}} \mu\right)\right] & & \text { (by lin. of } \left.\alpha_{i}\left[u_{i}\right](\cdot)\right) \\
& =\sum_{i=1}^{2} \alpha_{i}\left[u_{i}\right]\left(\left[\operatorname{Ex}_{\Omega_{i}} \mu\right)\right. & & \text { (by }(5)) \\
& =0 & & (\text { by }(7)) .
\end{aligned}
$$

Hence $u$ solves (2).

\section{Steklov-Poincaré Formulation}

Following the standard substructuring approach we now write the coupled problem (5)-(7) as a single equation on an interface space. In our setting this interface space is the nonlinear space $\Lambda$.

We first introduce the Steklov-Poincaré operators for the subdomain problems. For each subdomain, these map Dirichlet values on $\Gamma$ to the Neumann traces of the corresponding subdomain solutions on $\Gamma$. These Neumann traces are sections in a certain dual bundle. 
Definition 10. Let $u: \Omega \rightarrow M$ be continuous. For any Sobolev space $\Xi_{u}^{k, p}$ of sections in $u^{*} T M$ we call $\left(\Xi_{u}^{k, p}\right)^{*}$ its dual, i.e., the set of all linear functionals $L: \Xi_{u}^{k, p} \rightarrow \mathbb{R}$ such that $L(\eta)$ is finite for all $\eta \in \Xi_{u}^{k, p}$.

We denote by $\left(\Xi^{k, p}\right)^{*}$ the disjoint union of all spaces $\left(\Xi_{u}^{k, p}\right)^{*}$ for all continuous $u$. This concept allows to generalize the space $\left(H^{\frac{1}{2}}(\Gamma)\right)^{*}$ used for the Neumann traces of linear problems.

Definition 11. We call $S_{i}$ the Dirichlet-to-Neumann map associated to the $i$-th subdomain. That is, for any $\lambda \in \Lambda$ we set $S_{i} \lambda \in\left(\Xi_{\lambda}^{1 / 2}\right)^{*}$ to be such that

$$
S_{i} \lambda[\mu]=\alpha_{i}\left[u_{i}\right]\left(\operatorname{Ex}_{\Omega_{i}} \mu\right) \quad \text { for all } \mu \in \Xi_{\lambda}^{1 / 2},
$$

where $u_{i}$ fulfills $\operatorname{tr}_{\Gamma} u_{i}=\lambda$ and solves

$$
\alpha_{i}\left[u_{i}\right](\eta) \quad \text { for all } \eta \in V_{i, u_{i}}^{0} .
$$

Remark 1. We assume here for simplicity that the $S_{i}$ are single-valued, i.e., that for given Dirichlet data $\lambda$ the corresponding subdomain problems have unique solutions.

Using the Steklov-Poincaré operators we can write the coupled problem (5)-(7) as a problem on the interface space alone.

Lemma 7. The coupled problem (5)-(7) is equivalent to the Steklov-Poincaré equation

$$
S_{1} \lambda+S_{2} \lambda=0 .
$$

Note that $S_{1} \lambda$ and $S_{2} \lambda$ are from the same linear space $\left(\Xi_{\lambda}^{1 / 2}\right)^{*}$. Hence the addition is justified.

Proof. Let $\lambda \in \Lambda$. Then the subdomain solutions $u_{1}, u_{2}$ used in the definition of $S_{1}$ and $S_{2}$ solve the subdomain problems (5) by construction. Also, since they both assume the same value $\lambda$ on $\Gamma$ they are continuous on the interface. Finally, inserting (8) into (9) yields (7). Conversely, if $u_{1}, u_{2}$ solve (5)-(7), then $\lambda:=\operatorname{tr}_{\Gamma} u_{1}=\operatorname{tr}_{\Gamma} u_{2}$ solves (9).

\section{Nonlinear Preconditioned Richardson Iteration}

The natural algorithm for the Steklov-Poincaré interface equation (9) is the preconditioned Richardson iteration. Depending on the preconditioner, various different domain decomposition algorithms result, which we will describe below.

Let $k \in \mathbb{N}$ and $\lambda^{k} \in \Lambda$ be an iterate of the interface variable. Following Deparis et al. 3], we write one iteration of the preconditioned Richardson iteration in three steps: 
1. Compute residual $\sigma^{k} \in\left(\Xi_{\lambda^{k}}^{1 / 2}\right)^{*}$ by

$$
\sigma^{k}=S_{1} \lambda^{k}+S_{2} \lambda^{k}
$$

2. Get correction $v^{k} \in \Xi_{\lambda^{k}}^{1 / 2}$ by preconditioning the negative residual

$$
v^{k}=P_{\lambda^{k}}^{-1}\left(-\sigma^{k}\right)
$$

3. Do a damped geodesic update

$$
\lambda^{k+1}=\exp _{\lambda^{k}} \omega v^{k},
$$

where $\omega \in(0, \infty)$ is a parameter, and the map $\exp _{\lambda^{k}}$ is to be understood pointwise.

The preconditioner $P$ is a vector bundle morphism from $\Xi^{1 / 2}$ to $\left(\Xi^{1 / 2}\right)^{*}$, that is, a mapping from $\Xi^{1 / 2}$ to $\left(\Xi^{1 / 2}\right)^{*}$ such that $\pi(P v)=\pi v$ for all $v \in \Xi^{1 / 2}$, and such that for each $\lambda \in \Lambda$ the induced map from $\Xi_{\lambda}^{1 / 2}$ to $\left(\Xi_{\lambda}^{1 / 2}\right)^{*}$ is linear. It maps infinitesimal corrections to generalized stresses. We additionally require that each $P_{\lambda^{k}}$ be invertible. Consequently, its inverse $P_{\lambda^{k}}^{-1}$ maps generalized stresses to corrections.

The update step 3 needs to use the exponential map to apply the correction $v^{k}$ (which is a vector field along $\lambda^{k}$ ) to the current iterate $\lambda^{k}$. The correction is multiplied with a positive damping factor $\omega$. More generally, this factor can be replaced by a linear map $\omega^{k}$ from the tangent space $\Xi_{\lambda^{k}}^{1 / 2}$ onto itself. If $M$ is a linear space the exponential map degenerates to the addition of its argument to $\lambda^{k}$.

Remark 2. The two subdomain solves needed for Step 1 of the Richardson iteration can be performed in parallel. Since Step 1 is by far the most costly part this parallelization leads to considerable performance gains.

To construct preconditioners we introduce the derivatives of the SteklovPoincaré operators. For $S_{i}: \Lambda \rightarrow\left(\Xi^{1 / 2}\right)^{*}$ we interpret the derivative at a $\lambda \in \Lambda$ as a linear map $S_{i}^{\prime}(\lambda)$ from $\Xi_{\lambda}^{1 / 2}$ to $\left(\Xi_{\lambda}^{1 / 2}\right)^{*}$.

Remark 3. This interpretation is most easily understood if we assume for a second that the space $\Lambda$ is smooth enough to form a Banach manifold. We can then write vector fields as elements of the tangent bundle $T \Lambda$. The SteklovPoincaré operator $S_{i}$ becomes a map $S_{i}: \Lambda \rightarrow T^{*} \Lambda$, and its derivative at $\lambda \in \Lambda$ is the linear map $S_{i}^{\prime}: T_{\lambda} \Lambda \rightarrow T_{S_{i} \lambda} T_{\lambda}^{*} \Lambda$. Since $T_{\lambda}^{*} \Lambda$ is a linear space we can identify $T_{S_{i} \lambda} T_{\lambda}^{*} \Lambda$ with $T_{\lambda}^{*} \Lambda$, and therefore interpret $S_{i}^{\prime}(\lambda)$ as a linear map from $T_{\lambda} \Lambda$ to $T_{\lambda}^{*} \Lambda$. This corresponds to a map from $\Xi_{\lambda}^{1 / 2}$ to $\left(\Xi_{\lambda}^{1 / 2}\right)^{*}$ if $\Lambda$ is not sufficiently smooth.

We now describe various preconditioners and the algorithms that result from them. 
- Dirichlet-Neumann Preconditioner: The simplest choice for a preconditioner is the inverse of the linearized Steklov-Poincare operator of one of the subproblems. We define the Dirichlet-Neumann preconditioner as

$$
P_{\mathrm{DN}, k}:=S_{1}^{\prime}\left[\lambda^{k}\right] .
$$

With this choice, the damped preconditioned Richardson iteration reads

$$
\lambda^{k+1}=\exp _{\lambda^{k}}\left(\omega P_{\mathrm{DN}, k}^{-1}\left(-\sigma^{k}\right)\right)=\exp _{\lambda^{k}}\left[\omega\left(S_{1}^{\prime}\left[\lambda^{k}\right]\right)^{-1}\left(-S_{1} \lambda^{k}-S_{2} \lambda^{k}\right)\right] .
$$

Using instead the second subdomain for preconditioning we define the NeumannDirichlet preconditioner

$$
P_{\mathrm{ND}, k}:=S_{2}^{\prime}\left[\lambda^{k}\right] .
$$

- Neumann-Neumann Preconditioner: We can generalize the above construction by allowing arbitrary convex combinations of the Dirichlet-Neumann and Neumann-Dirichlet preconditioners. Let $\gamma_{1}, \gamma_{2}$ be two non-negative real numbers with $\gamma_{1}+\gamma_{2}>0$. Then

$$
P_{\mathrm{NN}, k}^{-1}:=\gamma_{1}\left(S_{1}^{\prime}\left[\lambda^{k}\right]\right)^{-1}+\gamma_{2}\left(S_{2}^{\prime}\left[\lambda^{k}\right]\right)^{-1}
$$

is the Neumann-Neumann preconditioner. When $M$ is a linear space and the equation to be solved is linear, then the Richardson iteration together with the preconditioner (10) reduces to the usual Neumann-Neumann iterative scheme.

- Robin Preconditioner: Finally, we generalize the Robin-Robin method. Let again $\gamma_{1}$ and $\gamma_{2}$ be two non-negative coefficients such that $\gamma_{1}+\gamma_{2}>0$. Further, let $F$ be a vector bundle morphism from $\Xi^{1 / 2}$ to $\left(\Xi^{1 / 2}\right)^{*}$ that is invertible on each fiber. For each $\lambda^{k} \in \Lambda, F_{\lambda^{k}}$ is a linear map from $\Xi_{\lambda^{k}}^{1 / 2}$ to $\left(\Xi_{\lambda^{k}}^{1 / 2}\right)^{*}$. We then define the Robin-Robin preconditioner

$$
P_{\mathrm{RR}, k}:=\frac{1}{\gamma_{1}+\gamma_{2}}\left[\gamma_{1} F_{\lambda^{k}}+S_{1}^{\prime}\left(\lambda^{k}\right)\right] F_{\lambda^{k}}^{-1}\left[\gamma_{2} F_{\lambda^{k}}+S_{2}^{\prime}\left(\lambda^{k}\right)\right] .
$$

For the linear finite-dimensional case, the identity map can be chosen for $F$. In that case the equivalence of this preconditioner to the Robin-Robin iterative method has been shown in 4 .

\section{Numerical Results}

We demonstrate the performance of the Richardson iteration with a numerical example. Consider a hyperelastic Cosserat shell. Configurations of such a shell are pairs of functions $(\varphi, R): \Omega \rightarrow \mathbb{R}^{3} \times \mathrm{SO}(3)$, where $\Omega$ is a twodimensional domain, and $\mathrm{SO}(3)$ is the set of orthogonal $3 \times 3$-matrices $R$ with $\operatorname{det} R=1$. For $x \in \Omega$ we interpret $\varphi(x) \in \mathbb{R}^{3}$ as the position of a point of 
the shell midsurface, and $R_{3}(x) \in \mathbb{R}^{3}$ (the third column of $R(x) \in \mathrm{SO}(3)$ ) as a transverse direction. The remaining two orthonormal vectors $R_{1}$ and $R_{2}$ describe an in-plane rotation (Figure 1). This choice of kinematics allows to model size-effects and microstructure. We use a hyperelastic material with the energy functional proposed by Neff in [8, Chap. 7]. For this energy, existence and partial regularity of minimizers have been shown [8, but no further analytical results are available.

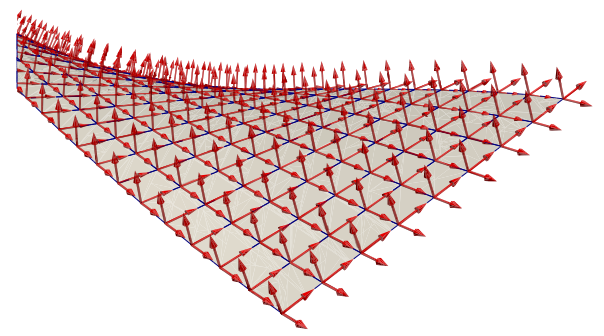

Fig. 1 Cosserat shell configurations consist of the deformation field $\varphi$ of the mid-surface, and an orientation field $R$ which can be interpreted as a field of three orthogonal director vectors.

As an example problem we use a rectangular strip of dimensions $10 \mathrm{~mm} \times$ $1 \mathrm{~mm}$. The thickness parameter is set to $0.05 \mathrm{~mm}$. Both the displacement $\varphi$ and the orientation $R$ are clamped at one of the short ends. At the other short end we prescribe a time-dependent Dirichlet boundary condition to the midsurface position $\varphi$ and rotations $R$, which describes a uniform rotation from 0 to $4 \pi$ about the long central axis of the strip. The positions and rotations at the long sides are left free. This makes the strip coil up. Note that we need the hyperelastic shell energy with the nonlinear membrane term proposed in Chapter 7 of [8] for this to work, because it is a finite strain example.

For the material parameters we choose the Lamé constants $\mu=3.8462$. $10^{5} \mathrm{~N} / \mathrm{mm}^{2}, \lambda=2.7149 \cdot 10^{5} \mathrm{~N} / \mathrm{mm}^{2}$, and the Cosserat couple modulus $\mu_{c}=$ $0 \mathrm{~N} / \mathrm{mm}^{2}$. The internal length scale is set to $L_{c}=0.1 \mathrm{~mm}$, and the curvature exponent is $p=1$ (see [8] for details on these parameters).

We divide the domain into two subdomains of dimensions $5 \mathrm{~mm} \times 1 \mathrm{~mm}$, and the time interval in 20 uniform time steps. For each time step we solve the spatial problem with a nonlinear Richardson iteration and the NeumannNeumann preconditioner of Section 4 , with $\gamma_{1}=\gamma_{2}=\frac{1}{2}$. The subdomain problems are discretized using first-order geodesic finite elements [11] on a uniform grid with quadrilateral elements, and the resulting nonlinear algebraic minimization problems are solved using a Riemannian trust-region algorithm [1, 11]. The linear preconditioner problems are solved using a CG method. The code was implemented on top of the Dune libraries [2]. 

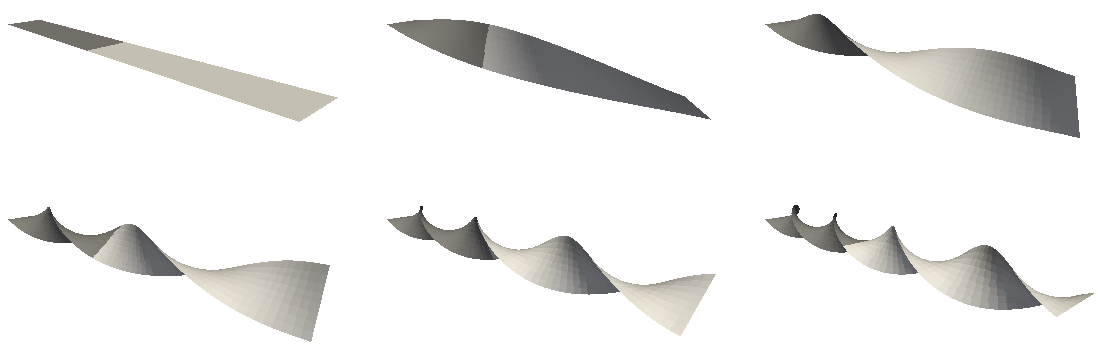

Fig. 2 Twisted elastic strip at rotation angles $0, \frac{4}{5} \pi, \frac{8}{5} \pi, \frac{12}{5} \pi, \frac{16}{5} \pi$, and $4 \pi$

Figure 2 shows several snapshots from the evolution of the strip. One can see how the strip coils up following the rotation prescribed to the boundary.
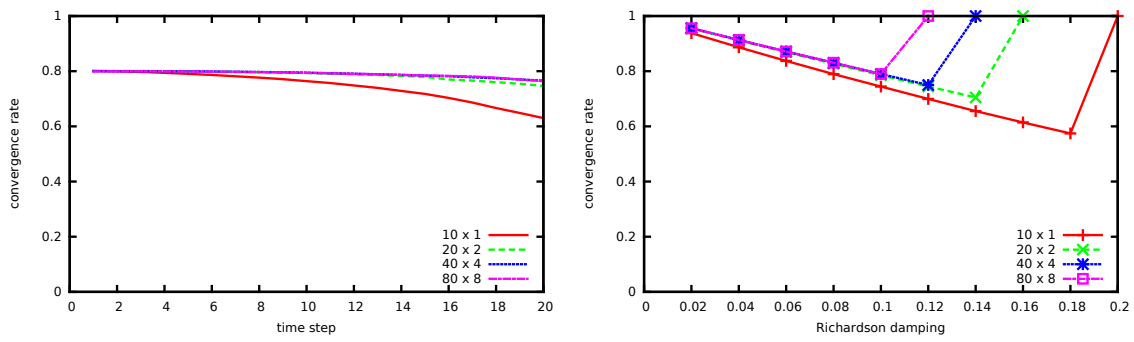

Fig. 3 Left: Convergence rates as a function of time for the Richardson damping parameter $\omega=0.1$, and different grid resolutions. Right: Convergence rates averaged over time, for several grid resolutions and values of $\omega$

To assess the convergence speed of the substructuring method we monitor the traces $\lambda^{k}$ defined on the interface $\Gamma=\{5\} \times[0,1]$. We estimate the convergence rate of the Neumann-Neumann solver at iteration $k$ by $\rho^{k}:=$ $\left\|v^{k}\right\| /\left\|v^{k-1}\right\|$, where $v^{k-1}$ and $v^{k}$ are two consecutive corrections produced by the Richardson iteration. For the norm $\|\cdot\|$ we use the Sobolev norm $H^{1}\left(\Gamma, \mathbb{R}^{3} \times \mathbb{R}^{4}\right)$, using the canonical embedding of $\mathrm{SO}(3)$ into the quaternions to embed tangent vectors of $\mathrm{SO}(3)$ into $\mathbb{R}^{4}$. This norm is well-defined for discrete functions. We let the domain decomposition algorithm iterate until the $H^{1}$-norm of the correction drops below $10^{-3}$. The overall convergence rate for one time step is then determined by taking the geometric average over the $\rho^{k}$.

We measure the rates as a function of the grid resolution and of the Richardson damping parameter $\omega$. One observes immediately that a rather small value for $\omega$ is needed to make the algorithm converge. Figure 3, left, shows the convergence rates for $\omega=0.1$ and four different grids as a function 
of time. Grid resolutions range from $10 \times 1$ to $80 \times 8$, created by uniform refinement. We see that the convergence rate is rather independent of the time step and of the grid resolution, with the exception of the coarsest grid, for which convergence rates ameliorate over time.

To get a better idea of the dependence of the convergence speed on the damping parameter $\omega$ we therefore average the rates over time and plot the results in Figure 3, right. We observe that the optimal $\omega$ decreases and the the optimal convergence rate increases as the grid is refined. This matches what is known for the linear case. A more detailed study of the behavior at vanishing mesh sizes, along with a proof of convergence, however, has to be left for future work.

\section{References}

[1] P.-A. Absil, R. Mahony, and R. Sepulchre. Optimization Algorithms on Matrix Manifolds. Princeton University Press, 2008.

[2] P. Bastian, M. Blatt, A. Dedner, C. Engwer, R. Klöfkorn, R. Kornhuber, M. Ohlberger, and O. Sander. A generic grid interface for parallel and adaptive scientific computing. Part II: Implementation and tests in DUNE. Computing, 82(2-3):121-138, 2008.

[3] S. Deparis, M. Discacciati, G. Fourestey, and A. Quarteroni. Fluidstructure algorithms based on Steklov-Poincaré operators. Comput. Methods Appl. Mech. Engrg., 195:5797-5812, 2006.

[4] M. Discacciati. An operator-splitting approach to non-overlapping domain decomposition methods. Technical Report 14.2004, Ecole Polytechnique Fédérale de Lausanne, 2004.

[5] N. Große and C. Schneider. Sobolev spaces on Riemannian manifolds with bounded geometry: General coordinates and traces. Mathematische Nachrichten, 286(16):1586-1613, 2013. arXiv 1301.2539v1.

[6] J. Jost. Riemannian Geometry and Geometric Analysis. Springer, 2011.

[7] N. J. Korevaar and R. M. Schoen. Sobolev spaces and harmonic maps for metric space targets. Comm. Anal. Geom., 1(4):561-659, 1993.

[8] P. Neff. A geometrically exact planar Cosserat shell-model with microstructure: Existence of minimizers for zero Cosserat couple modulus. Math. Mod. Meth. Appl. Sci., 17(3):363-392, 2007.

[9] R. S. Palais. Foundations of global non-linear analysis, volume 196. Benjamin New York, 1968.

[10] A. Quarteroni and A. Valli. Domain Decomposition Methods for Partial Differential Equations. Oxford Science Publications, 1999.

[11] O. Sander. Geodesic finite elements on simplicial grids. Int. J. Num. Meth. Eng., 92(12):999-1025, 2012. 